\title{
Impact on Public Health of Urinary Tract Catheters (UTCs) Using as Microbial Borne Infections due to Biofilms Composition, Taif, KSA
}

\author{
${ }^{* 1}$ Sherifa Mostafa M. Sabra, ${ }^{2}$ Abd-Allah D. Al-Talhi, ${ }^{3}$ Monuir M. Al-Bashan \\ ${ }^{*}{ }_{1,2} \& 3$ (Microbiology Br., Biology Dept., Science College, Taif University, KSA)
}

\begin{abstract}
This work was done in 2014 at Taif, KSA, patients (Pts.) for study male hospital admitted were treated by urinary tract catheters (UTCS). They were (No. $=50$ Pts.) normal and (No. $=50$ Pts.) with diseases. Incidence of biofilms presence on UTCs for short and long-terms, through short-term it were 30, and $42 \%$, and in long-term were 72, and 80\% for normal and diseased Pts. respectively. Incidence of biofilms presence on UTCs every other day during 4 Wks., its appeared increases incidence of biofilms presence on UTCs by: $(5 \pm 2 \%)$

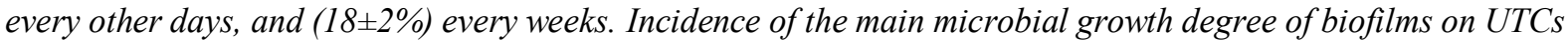
segments, in short-term were (normal Pts. 25, 25, 00, and 00\%) and (Diseased Pts. 50, 50, 25, and 00\%) for $1^{\text {st }}$, $2^{\text {nd }}, 3^{\text {rd }}$, and $4^{\text {th }}$ segments. In long-term were (normal Pts. 75, 50, 25, and 25\%) and (diseased Pts. 100, 100, 75, and $50 \%)$ for $1^{\text {st }}, 2^{\text {nd }}, 3^{\text {rd }}$, and $4^{\text {th }}$ segments respectively. Incidence the main and types of isolated microorganisms (MOs) from UTCs biofilms, MOs were isolated in short-term as 21, 17, 16, 15, 14, 13, 11, and 5\%, while in long-term were 45, 32, 26, 23, 22, 20, 19, and 12\% for E. coli, Candida albicans, Staph epidermidis, Klebsiella pneumoniae, Pseudomonas aeruginosa, Staph. aureus, Enterococcus faecalis and Proteus vulgaris respectively. The most efficient methods to prevent infections from UTCs biofilms are to avoid unnecessary catheterizations and to remove catheters as soon as possible. The use of hydrophilic-coated catheters for clean intermittent catheterization can effectively reduce microbial borne infections.
\end{abstract}

Keywords: Patients (Pts.), Urinary tract catheters (UTCs), Biofilms, Short-term, Long-term, Segments, Microorganisms (MOs).

\section{Introduction:}

A biofilm is an aggregate of MOs in which cells adhere to each other on a surface. These adherent cells are frequently embedded within a self-produced matrix of extracellular polymeric substance (EPS), is also referred to as slime, polymeric conglomeration generally composed of extracellular DNA, proteins, and polysaccharides. Its form on living or non-living surfaces and can be prevalent in hospital settings. MOs growing in a biofilm are physiologically distinct from cells of the same MO, which, by contrast, are single-cells, response to many factors, which may inhibitory concentrations of antibiotics ${ }^{[1]}$. Infectious processes was $80 \%$ biofilms had been implicated include common problems such as urinary tract infections (UTIs), catheter infections, middle-ear infections, formation of dental plaque, gingivitis, coating contact lenses ${ }^{[2]}$. Catheterassociated urinary tract infections (CAUTI) remain the most common nosocomial infection. Although usually benign, caused bacteremia in $(2-4 \%)$, and had been associated with a case fatality rate 3times as high. In persons with long-term catheterization, bacteriuria inevitably developed. Proteus produced catheter encrustations and persistent bacteriuria. Growth of MOs in biofilms on the inner surface of catheters promoted encrustation and may protected from antimicrobial agents ${ }^{[3]}$, it's may ascend the inner lumen into the patient's bladder in (1-3) days ${ }^{[4]}$. UTCs when inserted may readily acquire biofilms on the inner or outer surfaces. MOs commonly contaminating are Staph. epidermidis, Enterococcus faecalis, E. coli, Proteus mirabilis, Pseudomonas aeruginosa, Klebsiella pneumoniae, and other Gram-negative organisms ${ }^{[5]}$. UTC remains longer in place, the greater tendency of these MOs to develop biofilms and result in UTIs, $10 \%-50 \%$ of patients undergoing shortterm urinary catheterization (7days) but virtually all patients undergoing long-term catheterization ( $>28$ days) become infected ${ }^{[6]}$. Most CAUTIs are endogenous, that was, from the patient's own colonic flora. The indwelling catheter offers conducted to MOs entry along its external and internal surfaces. Uro-epithelial cells of catheterized patients caught transiently allow greater numbers of bacteria to adhere to their surfaces, a phenomenon that may precede the onset of bacteriuria ${ }^{[7]}$. The most frequent complication associated with catheters was the development of nosocomial CAUTIs, up to $50 \%$ of patients would at some point during their hospitalization been catheterized, severely increasing the patient's risk of colonization by $\mathrm{MO}$, which occurred in $(5-10 \%)$ of the patient's at each day of catheterization, Klebsiella pneumoniae, an important opportunistic pathogen frequently causing UTIs, septicemia, pneumonia in immune-compromised individuals, is responsible for up to $10 \%$ of all nosocomial bacterial infections ${ }^{[8]}$. The biofilm mode of growth had been implicated in the majority of human bacterial infections. UT is notable biofilm-associated infections included prostatitis, chronic 
cystitis, and CAUTIs ${ }^{[9]}$. MOs commonly attached to living and nonliving surfaces, including those of indwelling medical devices, and form biofilms made up of extracellular polymers ${ }^{[10]}$. Candida can cause both superficial and serious systemic disease, and were recognized as major agents of hospital-acquired infection, involved the formation of biofilms on implanted devices such as indwelling catheters ${ }^{[11]}$. Biofilm on UTCs resulted in persistent infections that were resistant to anti-microbial therapy. Because chronic catheterization leaded almost inevitably to bacteriuria ${ }^{[12]}$. The number of indwelling medical devices was escalating, and an increasing proportion of device-related infections were being caused by Candida spp., which produced biofilms on synthetic materials, facilitated adhesion of MOs to devices and rendered them relatively refractory to medical therapy. However, since the pathogenesis of Candida blood stream infection was complicated ${ }^{[13]}$. UT environment is the development of encrustation and consecutive obstruction. Modification of the biomaterial surface seemed to be the most promising prevention strategy for bacterial biofilms ${ }^{[14]}$. Many Candida albicans disease states involved biofilm growth. These infections had great impact on public health because MOs in biofilms exhibit dramatically reduced susceptibility to anti-fungal therapy. Progression to a mature biofilm was dependent on cell adhesion, extracellular matrix production and the yeast-to-hyphae transition ${ }^{[15]}$. Although the exact contribution of the catheter biofilm to pathogenicity was often unknown, the common recurrence of CAUTI soon after the completion of a course of antibiotic treatment was thought to be caused by re-colonization of urine by MOs that had survived in the catheter biofilm ${ }^{[16]}$. Using UTC was infected (21-50\%) of patients, the etiological agents were E. coli, Proteus mirabilis, Staph. aureus, Klebsiella spp., Pseudomonas spp., and Enterobacter spp. ${ }^{[17]}$. Presence of indwelling UTCs favored biofilm formation of Klebsiella pneumoniae and E. coli by providing an inert surface for the attachment of bacterial adhesins, thereby enhancing MOs colonization and the development of biofilm ${ }^{[18]}$. Biofilm development in UTCs was form of infection leaded to high mortality rates and causes significant costs in health care. The infection with uro-MOs like Pseudomonas aeruginosa ${ }^{[19]}$, Proteus mirabilis produced biofilms in diverse habitats with those formed in the human host playing a key role in indwelling device infections, were those formed when the MO is grown in urine, resulting in unique features including the presence of swarmer cells and hydroxyapatite crystals upon growth in urine. Factors relevant to biofilm formation included adhesion factors ${ }^{[20]}$. Adhesion to medical devices and biofilm formation were considered important virulence factors of Candida tropicalis, able to adhere and to form biofilms along the entire length of the catheters under flow conditions ${ }^{[21]}$. CAUTI was the most common health-care-associated infection worldwide $^{[22]}$. Microbial analysis of the intraluminal surface of the catheter is required for true identification of the causative agents of CAUTIs, allows for the complete characterization of biofilm-associated MOs species ${ }^{[23]}$. CAUTI was the most common device-associated healthcare-acquired infection. Long-term indwelling catheters were associated with more complex microbiology and greater morbidity than short-term catheters ${ }^{[24]}$. More than $40 \%$ of nosocomial infections were those of UT, most of these occurring in catheterized patients. Bacterial colonization on UTCs results various complications. The diversity of the biofilm microbial community increased with duration of catheter emplacement. One of the most important MO was Proteus mirabilis ${ }^{[25]}$. Biofilm producing bacteria as Staph. spp. and E. coli are cause of CAUTIs ${ }^{[26]}$. UTC is the most commonly deployed prosthetic medical device. It provides easy access to the bladder for bacteria contaminating the skin insertion site, CAUTIs are the most common infections acquired by patients in healthcare facilities. Bacterial biofilms form readily on these catheters and play important roles in the pathogenesis of the conditions that complicate the care and seriously threaten the health of catheterized patients ${ }^{[27]}$. Prolonged catheterization is the most important risk factor. As is the case with all device-related infections, the biofilm plays a central role in the pathogenesis of CAUTIs. The most effective way to prevent infection is to limit catheter use and discontinue the catheter when no longer needed ${ }^{[28]}$. A technology to prevent CAUTIs is still not available, however, there are promising results with new approaches such as the use of reminder systems and infection control programs, which can effectively decrease the rate of CAUTIs. There is evidence supporting the use of hydrophilic coated catheters for clean intermittent catheterization, but an optimal catheter material or coating still has to be developed. Evidence-based catheter management is crucial for every patient in need of a catheter ${ }^{[29]}$. Guidelines for prevention of CAUTIs should be followed, the most important of these is to avoid use of UTC whenever possible and, when there is no longer an indication for the catheter, to remove it promptly ${ }^{[30]}$.

The aim: Follow-up microbial biofilms formation on the inner surface of UTCs. UTCs always use for short and long-term which is the main source of microbial borne infections as CAUTIs is the second most common cause of nosocomial blood stream infection and other infections. This search summarizes the inner surface UTCs biofilms microbial contents according to the use period and patients conditions as a cause of microbial borne infections and affect the public health.

\section{Materials and Methods:}

Field for study: This work had done in 2014, at Taif, KSA, firstly selection of private hospitals, for research and had gotten consent forms from hospitals owners and urologists. 
Patients under study: Hospital admitted male pts., were treated by UTCs, they were (No. $=50 \mathrm{Pts}$.) normal and (No.=50Pts.) with diseases. Collection of consent forms from Pts. themselves for research work without any recording of personal information.

UTCs collection and preparation: UTCs specimens were collected under aseptic condition from male Pts., and labeled for (fixed and removal times of UTCs), then, transferred collected UTCs in sterile containers to microbiology laboratory, and refrigerated until processing, within $1 \mathrm{hr}$. UTCs were cut into 4axial segments each one is $10 \mathrm{~cm}$ diameter. The $1^{\text {st }}$ part is the most proximal 'bladder' section included the inserted tip of the catheter and the associated balloon. $2^{\text {nd }}, 3^{\text {rd }}$ were subsequent $10 \mathrm{~cm}$ segments comprising the 'deep urethral' and 'superficial urethral' regions, $4^{\text {th }}$ segment was an additional 'external' segment.

Microbial isolation and identification: Emersion of each segment in a separate Phosphate buffer solution (PBS) tube and agitate the tube for 30mintes to remove UTCs inner surface biofilms. Suspensions were resulted from PBS tubes were used for quantitative culture on Tryptic Soy agar, blood agar and Sabroud agar. The plates were incubated at $37^{\circ} \mathrm{C}$ for 2days, identification of colonies MOs types were carried by Micro-scan System ${ }^{[31]}$.

Data analysis: The data were recorded and entered into Microsoft Excel Sheet, then summarized and analyzed $^{[32]}$.

\section{Results and discussion:}

Table and diagram 1: Incidence of biofilms presence on *UTCs for short and long-terms

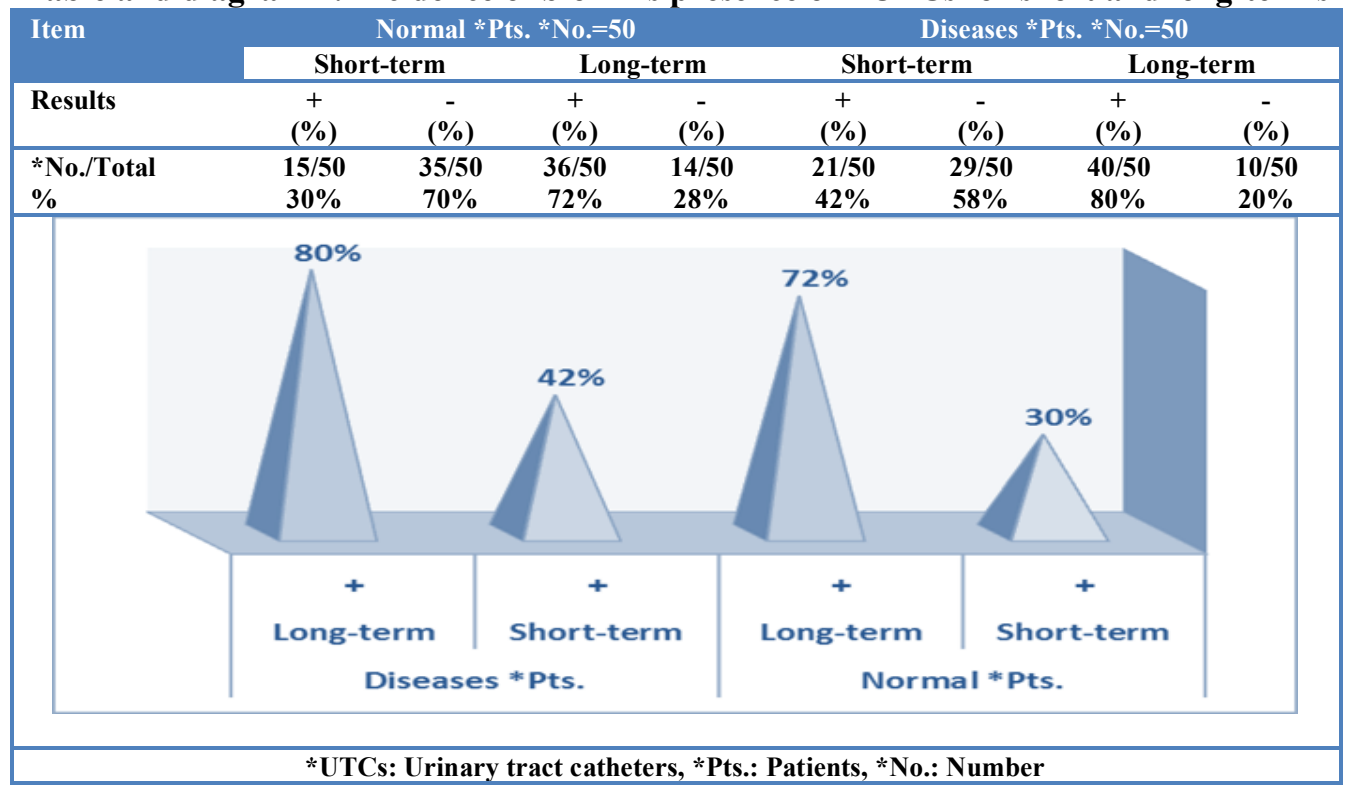

Table and diagram 1show incidence of biofilms presence on UTCs for short and long-terms, through short-term it were 30, and $42 \%$, and in long-term were 72 , and $80 \%$ for normal and diseased Pts. respectively. Growth of MOs in biofilms on the inner surface of catheters promoted encrustation and may protected from anti-microbial agents ${ }^{[3]}$. MOs may ascend the inner lumen into the patient's bladder in (1-3) days ${ }^{[4]}$. UTCs when inserted may readily acquire biofilms on the inner or outer surfaces ${ }^{[5]}$. UTC remains longer in place, the greater tendency of these MOs to develop biofilms and result in UTIs, 10\%-50\% of patients undergoing short-term urinary catheterization (7days) but virtually all patients undergoing long-term catheterization ( $>28$ days) become infected $^{[6]}$. MOs commonly attached to living and nonliving surfaces, including those of indwelling medical devices, and form biofilms made up of extracellular polymers ${ }^{[10]}$. Adhesion to medical devices and biofilm formation were considered important virulence factors. It was able to adhere and form biofilms along the entire length of the catheters under flow conditions ${ }^{[21]}$. CAUTI was the most common device-associated healthcareacquired infection. Long-term indwelling catheters were associated with more complex microbiology and greater morbidity than short-term catheters ${ }^{[24]}$. 
Table and diagram 2: Incidence of biofilms presence on *UTCs every other day during $4 *$ Wks.

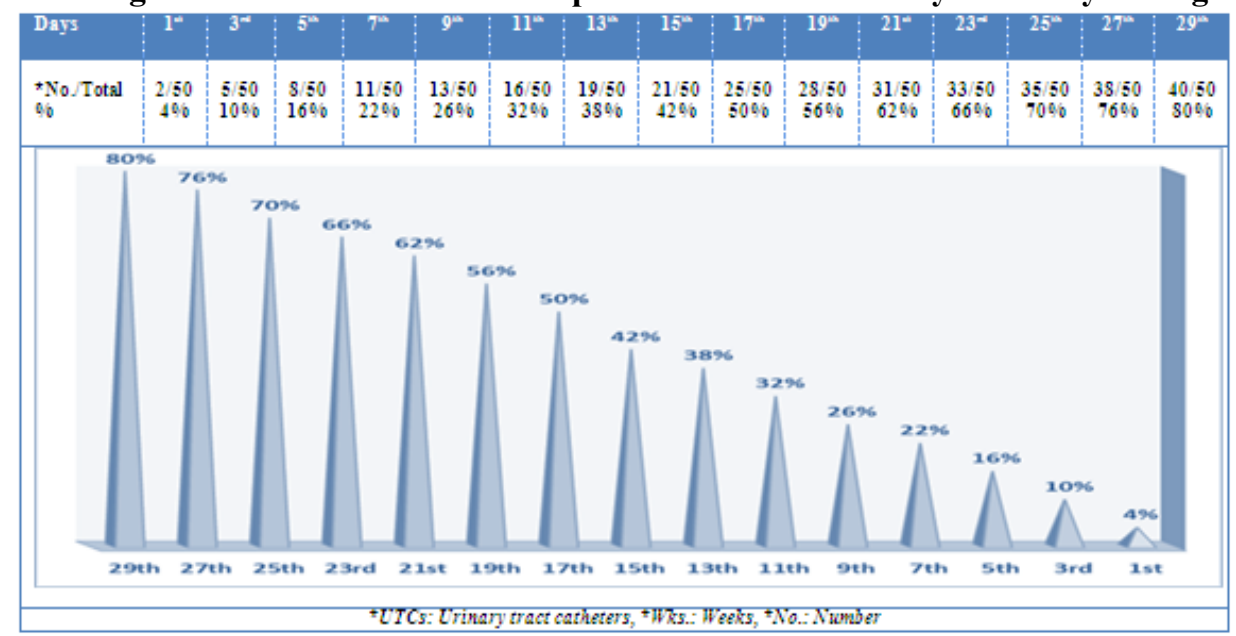

Table and diagram 2 show incidence of biofilms presence on UTCs every other day during 4Wks., its appeared increases incidence of biofilms presence on UTCs by: $(5 \pm 2 \%)$ every other days, and (18 $\pm 2 \%)$ every weeks. Biofilm on UTCs resulted in persistent infections that were resistant to anti-microbial therapy. Because chronic catheterization leaded almost inevitably to bacteriuria ${ }^{[12]}$. More than $40 \%$ of nosocomial infections were those of UT, most of these occurring in catheterized patients. Bacterial colonization of the UTCs results not only in infection, but also various complications. The diversity of the biofilm microbial community increased with duration of catheter emplacement ${ }^{[26]}$. UTC is the most commonly deployed prosthetic medical device. It provides easy access to the bladder for bacteria contaminating the skin insertion site. As a result, CAUTIs are the most common infections acquired by patients in healthcare facilities. Bacterial biofilms form readily on these catheters and play important roles in the pathogenesis of the conditions that complicate the care and seriously threaten the health of catheterized patients ${ }^{[27]}$. Prolonged catheterization is the most important risk factor. As is the case with all device-related infections, the biofilm plays a central role in the pathogenesis of infections ${ }^{[28]}$.

Table and diagram 3: Incidence of the main microbial growth degree of biofilms on *UTCs segments

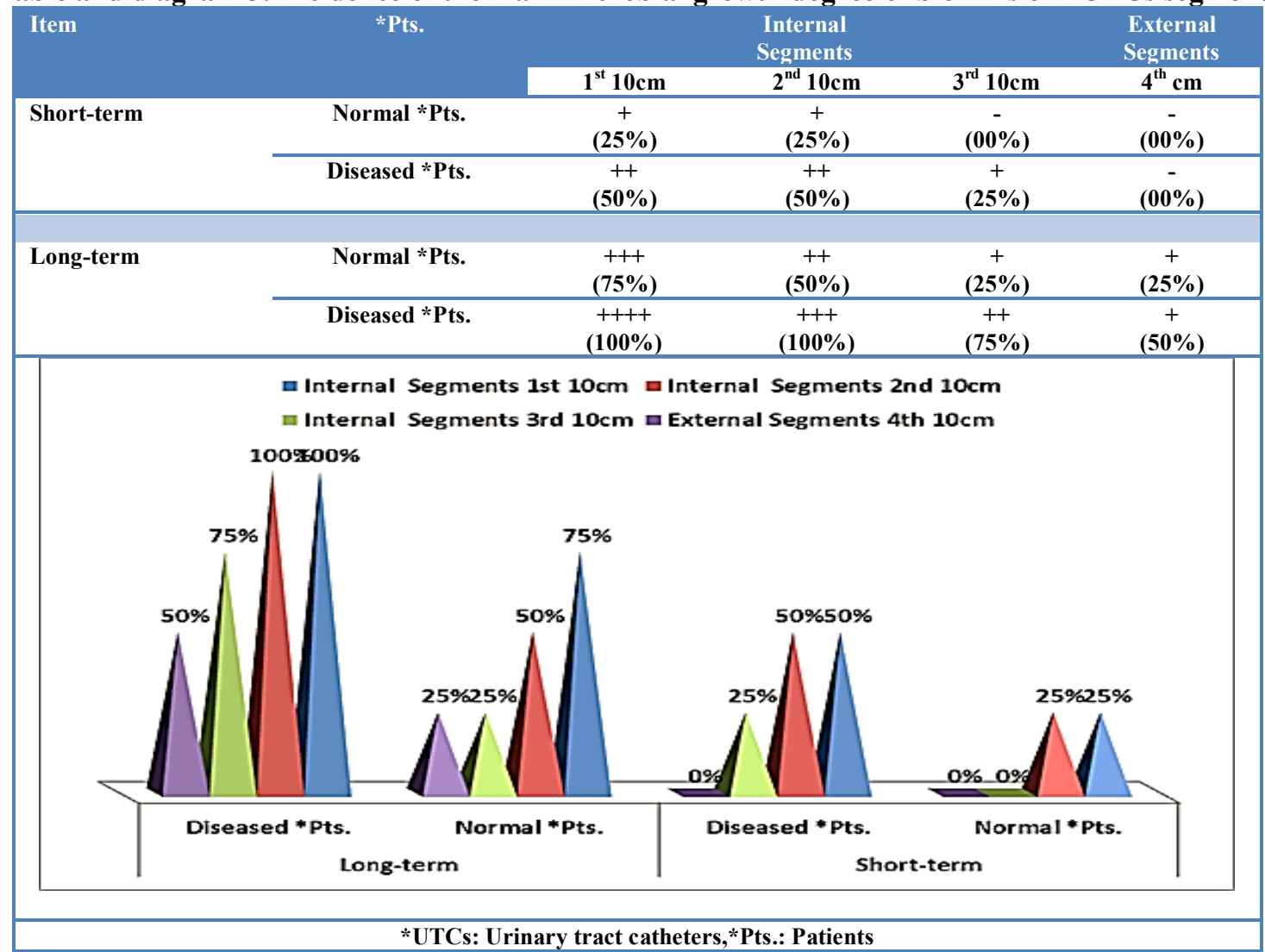


Table and diagram 3show incidence of the main microbial growth degree of biofilms on UTCs segments, in short-term were (normal Pts. 25, 25, 00, and 00\%) and (Diseased Pts. 50, 50, 25, and 00\%) for $1^{\text {st }}$, $2^{\text {nd }}, 3^{\text {rd }}$, and $4^{\text {th }}$ segments. In long-term were (normal Pts. 75, 50, 25, and 25\%) and (diseased Pts. 100, 100, 75, and $50 \%)$ for $1^{\text {st }}, 2^{\text {nd }}, 3^{\text {rd }}$, and $4^{\text {th }}$ segments respectively. The indwelling catheter offers conducted to MOs entry along its external and internal surfaces. Even with meticulous intentioned to maintenance of the closed system. Uro-epithelial cells of catheterized patients caught transiently allow greater numbers of bacteria to adhere to their surfaces, a phenomenon that may precede the onset of bacteriuria ${ }^{[7]}$. Up to $50 \%$ of patients would at some point during their hospitalization been catheterized, severely increasing the patient's risk of colonization by MO, which occurred in 5-10\% of the patient's at each day of catheterization, The biofilm mode of growth had been implicated in the majority of human bacterial infections. In the UT, notable biofilm-associated infections included prostatitis, chronic cystitis, and CAUTIs ${ }^{[9]}$.

Table and diagram 4: Incidence the main and types of isolated *MOs from *UTCs biofilms

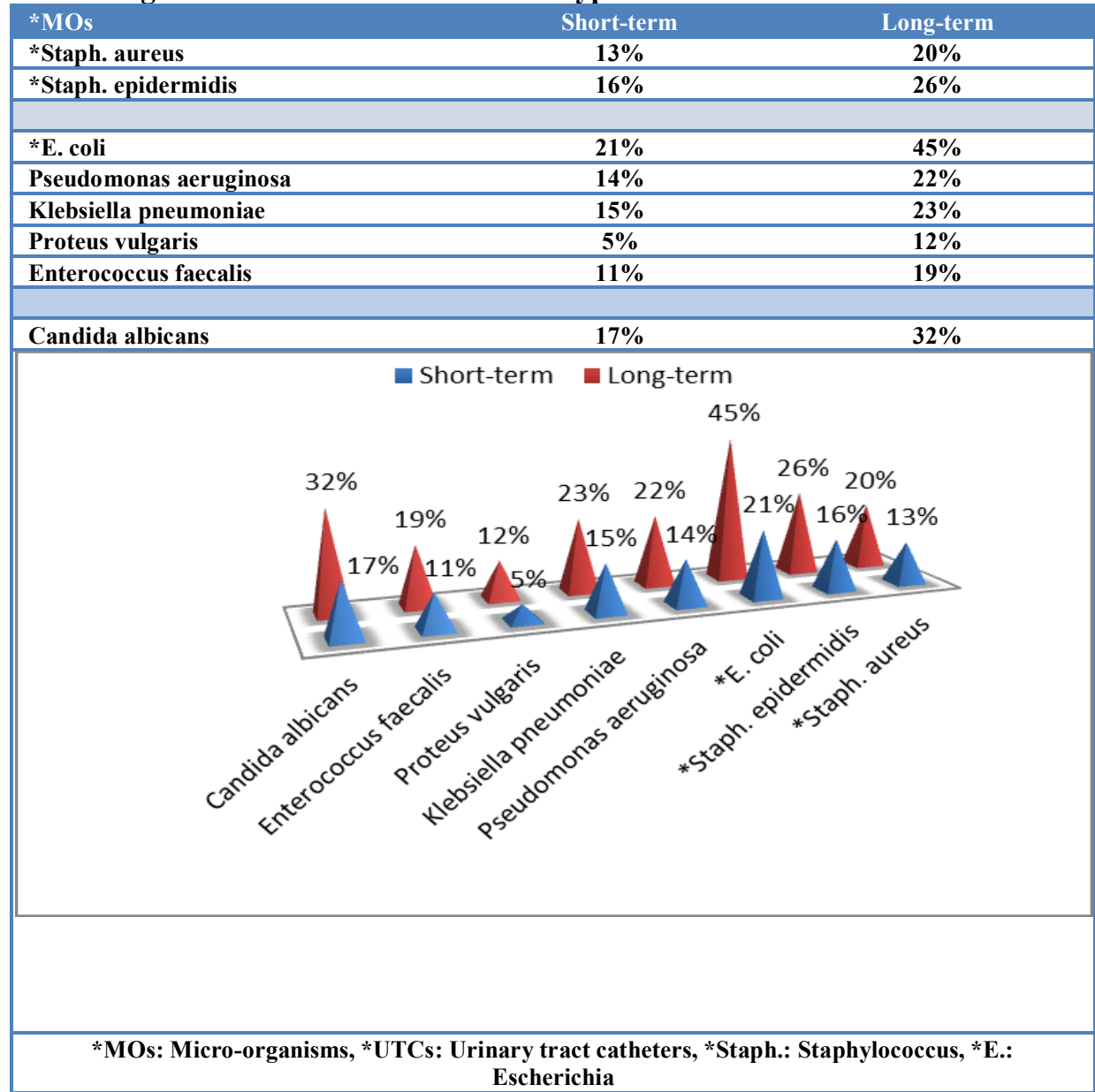

Table and diagram 4 show incidence the main and types of isolated MOs from UTCs biofilms, MOs were isolated in short-term as $21,17,16,15,14,13,11$, and 5\%, while in long-term were 45, 32, 26, 23, 22, 20, 19 , and $12 \%$ for E. coli, Candida albicans, Staph. epidermidis, Klebsiella pneumoniae, Pseudomonas aeruginosa, Staph. aureus, Enterococcus faecalis and Proteus vulgaris respectively. In persons with long-term catheterization, bacteriuria inevitably developed. Proteus produced catheter encrustations and persistent bacteriuria $^{[3]}$. MOs commonly contaminating are Staph. epidermidis, Enterococcus faecalis, E. coli, Proteus mirabilis, Pseudomonas aeruginosa, Klebsiella pneumoniae, and other Gram-negative organisms ${ }^{[5]}$. Klebsiella pneumoniae, an important opportunistic pathogen frequently causing UTIs, septicemia, pneumonia in immunecompromised individuals, is responsible for up to $10 \%$ of all nosocomial bacterial infections ${ }^{[8]}$. Pathogenic fungi Candida can cause both superficial and serious systemic disease, and were recognized as major agents of hospital-acquired infection, involved the formation of biofilms on implanted devices such as indwelling catheters $^{[11]}$. The number of indwelling medical devices was escalating, and an increasing proportion of devicerelated infections were being caused by Candida spp., which produced biofilms on synthetic materials, facilitated adhesion of the MOs to devices and rendered them relatively refractory to medical therapy. However, 
since the pathogenesis of Candida blood stream infection was complicated ${ }^{[13]}$. Many Candida albicans disease states involved biofilm growth. These infections had great impact on public health because MOS in biofilms exhibit dramatically reduced susceptibility to anti-fungal therapy. Progression to a mature biofilm was dependent on cell adhesion, extracellular matrix production and the yeast-to-hyphae transition ${ }^{[15]}$. Using UTC was infected $21-50 \%$ of patients, the etiological agents were E. coli, Proteus mirabilis, Staph. aureus, Klebsiella spp., Pseudomonas spp., and Enterobacter spp. ${ }^{[17]}$. Presence of indwelling UTCs favored biofilm formation of uro-pathogens as Klebsiella pneumoniae and E. coli by providing an inert surface for the attachment of bacterial adhesins, thereby enhancing MOs colonization and the development of biofilm ${ }^{[18]}$. The infection with uropathogenic MOs like Pseudomonas aeruginosa ${ }^{[19]}$, also, Proteus mirabilis produced biofilms in diverse habitats with those formed in the human host playing a key role in indwelling device infections, were those formed when the MO is grown in urine, resulting in unique features including the presence of swarmer cells and hydroxyapatite crystals upon growth in urine. Factors relevant to biofilm formation included adhesion factors ${ }^{[20]}$. Adhesion to medical devices and biofilm formation were considered important virulence factors of Candida tropicalis. It was able to adhere and to form biofilms along the entire length of the catheters under flow conditions $^{[21]}$. One of the most important pathogens in this regard was Proteus mirabilis ${ }^{[25]}$. Biofilm producing bacteria such as Staph. spp. and E. coli are the most common cause of CAUTIs ${ }^{[26]}$. Biofilm development in UTCs was form of infection leaded to high mortality rates and causes significant costs in health care CAUTI was the most common health-care-associated infection worldwide. Although not all cases of bacteriuria resulted in clinical infection ${ }^{[22]}$. A technology to prevent CAUTIs is still not available, however, there are promising results with new approaches such as the use of reminder systems and infection control programs. There is evidence supporting the use of hydrophilic coated catheters for clean intermittent catheterization ${ }^{[29]}$.

\section{Conclusions}

Microbial biofilms of UTCs may pose a public health problem for persons requiring indwelling medical devices. MOs in biofilms are difficult or impossible to treat with antimicrobial agents; detachment from the device may result in many infection. The most efficient methods to prevent microbial borne infections are to avoid unnecessary catheterizations and to remove catheters as soon as possible. The use of different reminder systems and implementation of infection control programs can effectively decrease UTCs bore infections. There is still no evidence to support the routine use of anti-microbial-impregnated catheters, but the use of hydrophiliccoated catheters for clean intermittent catheterization can effectively reduce borne infections. Preliminary results with Chlorhexidine-coated catheters are promising.

\section{Acknowledgements}

Thankful to hospital owners, urologists and Pts. who help in producing this paper.

\section{References:}

[1]. Karatan, E. and Watnick, P., 2009. Signals, regulatory networks, and materials that build and break bacterial biofilms. Micro. Molecular Bio. Rev., 73: 310-347.

[2]. Rogers, A., 2008. Molecular Oral Microbiology. Caister Acad. Press, PP.: 65-108.

[3]. Walter, E., 1991. Catheter-associated urinary tract infections: Epidemiology, pathogenesis, and prevention. Am. J. Med., 91: S65S71.

[4]. McLean, R., Nickel, J. and Olson, M., 1995. Biofilm associated urinary tract infections. In: Lappin-Scott, H., Costerton, J., editors. Microbial biofilms. Cambridge: Cambridge University Press., PP.: 261-273.

[5]. Stickler, D., 1996. Bacterial biofilms and the encrustation of urethral catheters. Biofouling., 94: 293-305.

[6]. Brisset, L., Vernet-Garnier, V., Carquin, J., Burde, A., Flament, J. and Choisy, C., 1996. In vivo and in vitro analysis of the ability of urinary catheters to microbial colonization. Pathol. Biol., 44: 397-404.

[7]. John, W., 1997. Catheter-associated urinary tract infections. Inf. Dis. Clinics of North America, 11: 609-622.

[8]. Podschun, R. and Ullmann, U., 1998. Klebsiella spp. as nosocomial pathogens: epidemiology, taxonomy, typing methods, and pathogenicity factors. Clin Micro. Rev., 11: 589-603.

[9]. Morris, N., Stickler, D. and Mclean, R., 1999. The development of bacterial biofilms on indwelling urethral catheters. World J. Uro., 17: 345-350.

[10]. Donlan, R., 2001. Biofilms and device-associated infections. Emerging Inf. Dis., 7:277-281.

[11]. Julia, L., 2003. Candida biofilms and their role in infection. Trend Micro., 11: 30-36.

[12]. Barbara, W. and Rabih, O., 2004. Role of biofilm in catheter-associated urinary tract infection. Am. J. Infect. Control, 32: 177-183.

[13]. Erna, M. and Rabih, O., 2004. Candida infections of medical devices. Cline, Micro. Rev., 17: $255-267$.

[14]. Tenke, P., Kovacs, B., Jackel, M. and Nagy, E., 2006. The role of biofilm infection in urology. World J. Ero., 24: 13-20.

[15]. Jeniel, N. and David, A., 2006. Candida albicans biofilm development, modeling a host-pathogen interaction. Curr. Op. Micro., 9: $340-345$.

[16]. Jacobsen, S., Stickler, D., Mobley, H. and Shirtliff, M., 2008. Complicated catheter-associated urinary tract infections due to Escherichia coli and Proteus mirabilis. Cline. Micro. Rev., 21: 26-59.

[17]. Omay, G., Jacobsen, S., Stickler, D., Mobley, H. and Shirtliff, M., 2009. Complicated urinary tract infections due to catheters. Springer, 3: 123-165.

[18]. Wang, X., Lunsdorf, H., Ehren, I., Brauner, A. and Romling, U., 2010. Characteristics of biofilms from urinary tract catheters and presence of biofilm-related components in E. coli. Curr. Micro., 60: 446-453. 
[19]. Katrin, D., Marie, S., Maren, M., Karin, A., Marina, W. and Petra, G., 2011. An in vitro-urinary tract catheter system to investigate biofilm development in catheter-associated urinary tract infections. J. Micro. Methods, 87: 302-308.

[20]. Sandra, M. and Mark, E., 2011. Proteus mirabilis biofilms and catheter-associated urinary tract infections. Virulence, 2: $460-465$.

[21]. Melyssa, N., Sonia, S., Mariana, H., Joana, A., Terezinha, S. and Rosario, O., 2011. Candida tropicalis biofilms: artificial urine, urinary catheters and flow model. Med. Myco., 49:739-747.

[22]. Danish, M. and Robih, O., 2012. New strategies to prevent catheter-associated urinary tract infections. Nature Rev. Uro., 9: 305314.

[23]. Ryad, D., Warda, B., Thierry, J. and Bouzid, M., 2012. Characterization of bacterial biofilms on urinary catheters. Am. J. Infect. Con., 31: 120-126.

[24]. Nicolle, L., 2012. Urinary catheter-associated infections. Infect. Dis. Cline. North Am., 26: 13-27.

[25]. Veronika, H., Tereza, P. and Filip, R., 2012. Virulence factors in Proteus bacteria from biofilm communities of catheter-associated urinary tract infections. FEMS Immun. and Med. Micro., 65: 343-349.

[26]. Kerry, L., Simon, A., Suzanne, W., David, C. and Navindra, P., 2012. Effects of Cranberry extracts on growth and biofilm production of E.coli and Staph. spp. Phyto-therapy Res., 26: 1371-1374.

[27]. David, S. and Roger, F., 2013. The Indwelling Bladder Catheter: Attempts to Prevent Infection and the Development of Bacterial Biofilms. Bio. Ass. Inf., Ch. 18: 455-483.

[28]. Mayar, A. and Rabih, O., 2013. Prevention and Treatment of Urinary Catheter-Associated Infections. Curr. Inf. Dis. Rep., 15: 116123.

[29]. Tenke, P., Köves, B. and Johansen, T., 2014. An update on prevention and treatment of catheter-associated urinary tract infections. Curr. Op. Inf. Dis., 27: 102-107.

[30]. Lindsay, E., 2014. Catheter-Related Urinary Tract Infection: Practical Management in the Elderly. Springer Drugs and Aging, 31: $1-10$.

[31]. MMWR., 2012. Central for Disease Control and Prevention. Guidelines for safe work practices in human and animal medical diagnostic laboratories, 61, Ch. (1,7).

[32]. Coulombier, D., Fagan, R., Hathcock, L. and Smith, C., 2001. Epi Info 6 Version 6.04.A Word Processing, Database and Statistical Program for Public Health. Centers for Disease Control and Prevention, Atlanta, Delaware, USA. 